PUPT-1354

TAUP-2013-92

hep-th/9212114

December 1992

\title{
GROUND STATE OF 2D QUANTUM GRAVITY AND SPECTRAL DENSITY OF RANDOM MATRICES
}

\author{
Marek Karliner * \\ Alexander Migdal ${ }^{\dagger}$ \\ Boris Rusakov* \\ * School of Physics and Astronomy \\ Raymond and Beverly Sackler Faculty of Exact Sciences \\ Tel-Aviv University, 69978 Tel-Aviv, Israel \\ $\dagger$ Physics Department and \\ Program in Applied and Computational Mathematics \\ Fine Hall, Princeton University \\ Princeton, NJ 08544-1000
}

\begin{abstract}
We compute the exact spectral density of random matrices in the ground state of the quantum hamiltonian corresponding to the matrix model whose double scaling limit describes pure gravity in $2 \mathrm{D}$. We show that the non-perturbative effects are very large and in certain cases dominate the semi-classical WKB contribution studied in the earlier literature. The physical observables in this model are the loop averages with respect to the spectral density. We compute their exact ground-state expectation values and show that they differ significantly from the values obtained in the WKB approximation. Unlike the alternative regularizations of the nonperturbative 2D quantum gravity, based on analytic continuation of the Painlevé transcendent, our solution shows no pathologies.
\end{abstract}


The discovery [1] of the double scaling limit of the matrix models [2] of 2D Gravity and its relation [3] to the $\mathrm{KdV}$ hierarchy opened the way to the study of the nonperturbative phenomena in Gravity and String theories. These phenomena are supposed to be described by the so called string equation

$$
x=\sum t_{l}(2 l+1) R_{l}[C]
$$

where $x$ is the scaling combination of cosmological constant and parameter $1 / N$ of the genus expansion, $t_{l}$ are the mass parameters of the theory and $R_{l}[C]=C^{l}+$ derivatives are the Gelfand-Dikii differential functionals corresponding to the specific heat $C(x)$ as the potential of the Schrödinger operator ( see e.g. [3] for details).

The string equation is in general a differential equation for $C(x)$ of the order corresponding to the highest $l$ in the sum. As observed in [4], for odd $l$ there is the natural boundary condition

$$
C(x) \rightarrow x^{1 / l} \quad x \rightarrow \pm \infty
$$

which uniquely determines the solution.

For the even $l$ case situation is more complicated. The above boundary condition would lead to a complex solution, corresponding analytic continuation of the divergent matrix integral [5]. The attempt to define the even $l$ case as the limit $t_{l+1} \rightarrow 0$ of the odd case [6] does not work, since the limit does not exist.

One way to interpret this paradox is to claim the even case and in particular the $l=2$ case of pure 2D Gravity inconsistent. That would be an exciting possibility : the nonperturbative creation of matter in Gravity. However, the matter here is not quite physical, as it does not correspond to the unitary multiplets of conformal field theory. The partition functions of the $l>2$ models with higher genus are not positive [7].

So, in fact, this interpretation implies that no 2D Gravity could exist at nonperturbative level. This is too serious a statement to make without investigating the alternative interpretations.

One such interpretation, suggested by Marinari and Parisi [8], along the lines of the general method by Greensite and Halpern [9], seems particularly appealing. They do not add any ad hoc terms to stabilize the equation, but rather modify the basic definition of the matrix model to make it meaningful for arbitrary potential. In Ref. [8] the spectral density of the resulting quantum hamiltonian was studied in the semi-classical WKB approximation. It turns out to be possible to go beyond the WKB approximation and to obtain the exact spectral density of this hamiltonian. The necessary framework was set up in Ref. [10], in which the set of equations generalizing the string equation was derived and studied numerically. Quantum effects, invisible in the WKB approximation, turned out to be extremely important.

The prescription of Refs. [8], [9] has attracted a considerable amount of attention, [11][21]. In particular, there have been some interesting attempts to study the properties of the model numerically at finite $N$. The problem encountered in these studies is the same as with all numerical studies of the matrix models, namely the double scaling limit requires very large $N$, because of the $N^{1 / 5}$ dependence of physical observables. 
The goal of the present paper is to apply the analytic and numerical methods of the note [10] to derive the exact spectral density of random matrices in the ground state of 2D Gravity. As a result, we are able to demonstrate that the non-perturbative effects are very large and in certain cases completely dominate the semi-classical WKB contribution. The physical observables in this model are the loop averages with respect to the spectral density. We compute their exact ground-state expectation values and show that they differ significantly from the values obtained in the WKB approximation.

We begin by discussing the method of Greensite and Halpern for the simplest example of a one dimensional integral. Consider a positive action $S(x)$ depending on a single variable $x$ :

$$
Z=\int d x \exp [-S(x)]
$$

By definition, the average of an operator $Q(x)$ in the action $S$ is given by

$$
\langle Q\rangle_{Z} \equiv \frac{\int d x Q(x) \exp [-S(x)]}{\int d x \exp [-S(x)]}
$$

If we define

$$
\psi_{0} \equiv \exp [-S(x) / 2] / \sqrt{Z}
$$

the expectation value (画) can be written

$$
\langle Q\rangle_{Z}=\left\langle\psi_{0}|Q| \psi_{0}\right\rangle
$$

$\psi_{0}$ is always positive and therefore it is always possible [22], [9], [8] to construct a quantummechanical hamiltonian $H=-d^{2} / d x^{2}+V(x)$, such that $\psi_{0}$ is its ground-state wave-function with the ground-state energy equal to zero:

$$
\begin{array}{r}
H \psi_{0}=0 \\
-\psi_{0}^{\prime \prime}(x)+V(x) \psi_{0}(x)=0 \\
V(x)=\psi_{0}^{\prime \prime}(x) / \psi_{0}(x)
\end{array}
$$

It is instructive to rewrite this hamiltonian in a manifestly positive form, as a product of an operator and its hermitean conjugate,

$$
H=\left(-\frac{d}{d x}+\frac{S^{\prime}(x)}{2}\right)\left(\frac{d}{d x}+\frac{S^{\prime}(x)}{2}\right)
$$

$\psi_{0}$ is annihilated by the second factor and hence $H \psi_{0}=0$. When the action $S$ is unbounded from below, the average (4) is ill-defined. Formally, (5) is still annihilated by (8), but $\psi_{0}$ is now not normalizable and therefore cannot be the ground state of $H$. On the other hand, $H$ is a positive hamiltonian by construction and therefore a normalizable ground state $\Psi_{0}$ must exist. Clearly the true ground state $\Psi_{0}$ must be different from $\psi_{0}$ and must have an eigenvalue $e_{0}>0$. Consider the action $S(x)=x^{2}-g^{2} x^{4}$. The average (4) is ill defined, but 
the power-series expansion in $g^{2}$ exists and can be computed explicitly. Each order in the expansion in powers of $g^{2}$ involves only moments of $x$ with a gaussian measure,

$$
\begin{array}{r}
\exp [-S(x)]=\exp \left(-x^{2}\right) \sum_{k=0}^{\infty} \frac{\left(g^{2} x^{4}\right)^{n}}{n !} \\
\langle Q\rangle_{Z}^{l}=\frac{\int d x Q(x) \exp \left(-x^{2}\right) \sum_{n=0}^{l}\left(g^{2} x^{4}\right)^{n} / n !}{\int d x \exp \left(-x^{2}\right) \sum_{n=0}^{l}\left(g^{2} x^{4}\right)^{n} / n !}
\end{array}
$$

Any sensible definition of average with the action $S(x)$ ought to reproduce the perturbation expansion (9) and it should reduce to (4) for a positive action. The quantum-mechanical expectation value in the true ground-state $\Psi_{0}$ of $H$ satisfies both constraints, we therefore take it as the definition of the average with the action $S$ :

$$
\langle Q\rangle_{Z}=\left\langle\Psi_{0}|Q| \Psi_{0}\right\rangle
$$

This prescription can be applied to matrix models,

$$
e^{F}=Z=\int d \phi \exp \left[-\beta \operatorname{tr} U_{k}(\phi)\right]
$$

where $\phi$ is a hermitean $N \times N$ matrix and the critical potentials are given by

$$
U_{k}(\phi)=\int_{0}^{1} \frac{d t}{t}\left[1-\left(1-t(1-t) \phi^{2}\right)^{k}\right]
$$

At large $\phi$ the $\phi^{2 k}$ term dominates, with the coefficient $(-1)^{k+1} \int_{0}^{1} d t t^{k-1}(1-t)^{k}$. Therefore the $k$-even critical potentials are unbounded from below and it is necessary to provide a prescription for defining the average (11) beyond perturbation expansion. Following the work of Greensite and Halpern [9] and Marinari and Parisi [8], we adopt the prescription (10). After all, the only existing justification of the matrix models as theories of gravity is perturbative, in the sense of the genus expansion. The quantum mechanical definition is a priori as good as the statistical one, but has the advantage of being guaranteed to make sense for all models. The extra bonus of this prescription is the relation to the one dimensional supersymmetric string theory [8], with dynamically broken supersymmetry in the even $l$ case. For practical purposes the supersymmetry seems to be useless so far, but one may hope to relate it to the physical supersymmetric string theories.

The simplest case of an unbounded potential in a matrix model is the cubic potential

$$
U(\phi)=\frac{\phi^{2}}{2}-g \frac{\phi^{3}}{3}
$$

In order to proceed, it is useful to change variables from the hermitean $N \times N$ matrices to their eigenvalues, $\left\{\lambda_{1}, \ldots, \lambda_{N}\right\}$ :

$$
e^{F}=Z=\int \prod_{i} d \lambda_{i} \prod_{i<j}\left(\lambda_{i}-\lambda_{j}\right)^{2} \exp [-\beta \operatorname{tr} U(\lambda)]
$$


The factor $\prod_{i<j}\left(\lambda_{i}-\lambda_{j}\right)^{2}$ comes from the Jacobian. In the following it is useful to include this factor in the wavefunction. The integration measure is then simply $\int \prod_{i} d \lambda_{i}$. It follows that the effective wave function corresponding to (14) is

$$
\Psi^{e f f}=\prod_{i<j}\left(\lambda_{i}-\lambda_{j}\right) \psi(\lambda) / \sqrt{Z}
$$

The hamiltonian $H$ is immediately obtained from (8) and (14):

$$
H=\sum_{i} H_{i} ; \quad H_{i}=-\frac{\partial^{2}}{\partial \lambda_{i}^{2}}+V_{\text {eff }}\left(\lambda_{i}\right) ; \quad V_{\text {eff }}(\lambda)=N \beta\left(g \lambda-\frac{1}{2}\right)+\frac{\beta^{2}}{4}\left(\lambda-g \lambda^{2}\right)^{2}
$$

(15) and (16) describe the ideal Fermi gas of $N$ non-interacting particles. The fermionic property is generic - it is due to the Jacobian of the transformation from the matrices $\phi$ to their eigenvalues. On the other hand, the fact that the effective fermions are free is only true when the original potential $U(\phi)$ is cubic in $\phi$.

The effective potential depends on the free variable $\lambda$ and on three parameters of the original matrix-model action: $N, \beta$ and $g, V_{\text {eff }}=V_{\text {eff }}(N, \beta, g ; \lambda)$. We are interested in the critical properties of the theory in the double scaling limit,

$$
x=\frac{\beta-N}{N^{1 / 5}} ; \quad N \rightarrow \infty ; \quad x \sim N^{0}
$$

with the critical point at $x=0$. For small positive $x$ the effective potential $V_{\text {eff }}(N, \beta, g ; \lambda)$ has a double-well shape. In the double scaling limit (17) the depth of the left well scales like $\sim N^{2}$, while the depth of the right well scales like $\sim x^{3 / 2} \sim N^{0}$. Despite this, it is the right, tiny well, which is responsible for all the interesting critical phenomena in the double scaling limit. All the cubic potentials of the type (13) are in the same universality class. At the critical point the depth of the second well goes to zero, and the potential has an inflection point, instead of a double minimum.

Since we are interested in having the critical point at $x=0$, it is necessary to chose a value of $g$ such that for $x=0$, i.e. for $\beta=N$, the extremum is an inflection point:

$$
\left.\frac{\partial V_{\text {eff }}}{\partial \lambda}\right|_{\lambda=\lambda_{0}}=0 ;\left.\quad \frac{\partial^{2} V_{\text {eff }}}{\partial \lambda^{2}}\right|_{\lambda=\lambda_{0}}=0
$$

leading to

$$
\begin{gathered}
g=\frac{1}{\sqrt{12 \sqrt{3}}} \\
\lambda_{0}=(\sqrt{3}+1) \sqrt[4]{3}
\end{gathered}
$$

We are interested in the scaling properties of the Fermi energy, or ground state, of the hamiltonian (16). The scaling properties are determined by the tiny right well, but the bulk of energy levels is in the left, bigger well. The Fermi energy, $e_{F}$ can be written as

$$
e_{F}=e_{F}^{0}+e_{F}^{s}(x)
$$


where the bulk part, $e_{F}^{0}$, is determined by the left well and $e_{F}^{s}(x)$ is the $x$-dependent scaling part that we are interested in. The depth of the left well $\sim N^{2}$, while the depth of the right well $\sim N^{0}$. In terms of magnitude, $e_{F}$ is completely dominated by $e_{F}^{0}$. The hamiltonian (16) can only be solved by some approximation procedure, analytical or numerical. It is mandatory to first isolate and "magnify" the scaling part, otherwise any approximate result for $e_{F}$ will be dominated by $e_{F}^{0}$ and the $x$ dependence of the fine-structure will be lost.

To isolate the scaling part, we expand the effective potential in $x$ and in $\lambda-\lambda_{0}$ around the inflection point and obtain the scaling potential $v(y)$ :

$$
\begin{gathered}
V_{\text {eff }}(N, \beta, g ; \lambda)=V_{\text {eff }}\left(N=\beta, g ; \lambda=\lambda_{0}\right)+\beta^{4 / 5} \alpha^{2 / 5} v(y) \\
v(y)=\frac{y^{3}}{3}-\epsilon y
\end{gathered}
$$

where $x$ is given by $(17)$ and

$$
y=\left(\lambda-\lambda_{0}\right) \alpha^{1 / 5} \beta^{2 / 5}, \quad \epsilon=x \alpha^{-3 / 5}, \quad \alpha=\frac{2+\sqrt{3}}{4 \sqrt[4]{3}}
$$

The critical properties of the theory are thus determined by the scaling hamiltonian

$$
h=-\frac{\partial^{2}}{\partial y^{2}}+v(y)
$$

The cubic potential $v(y)$ (not to be confused with the original cubic potential (13) ) is formally unbounded from below. This is not a problem, however, since $v(y)$ is only meaningful in the scaling region and the full potential $V_{\text {eff }}(\lambda)$ is bounded. The strategy for computing the fine structure of the Fermi energy is then as follows. An exact numerical solution for the spectral density of the scaling hamiltonian (25) can be obtained through the powerful methods of Gelfand and Dikii [24]. The exact solution can then be compared with the spectral density obtained from the WKB approximation. The two differ in the scaling region only, since WKB is completely adequate in the large left well. Their difference converges fast, yielding the contribution of the scaling region.

In order to obtain the density of states, we first write down the general equations of the Fermi-gas theory in one dimension. It is convenient to scale out $\beta$ from the Hamiltonian, and introduce the resolvent,

$$
G(e, y)=\left\langle y\left|(h-e)^{-1}\right| y\right\rangle
$$

The particle density $\rho(e, y)$ is related to the imaginary part of the resolvent,

$$
\rho(e, y)=\frac{1}{\pi} \operatorname{Im} G(e+i 0, y)
$$

and the spectral density $\nu(e)$ is given by the integral of $\rho$

$$
\nu(e)=\int_{-\infty}^{\infty} d y \rho(e, y) .
$$


The normalization of $\nu(e)$ is fixed by the fact that (15) and (16) describe $N$ non-interacting fermions. We rescale the spectral density by $N$ and the equation for the Fermi energy $e_{F}$ is therefore

$$
1=\int_{-\infty}^{e_{F}} d e \nu(e)
$$

The WKB particle density is given by

$$
\rho_{W K B}(e, y)=\frac{1}{2 \pi \sqrt{e-v(y)}}
$$

with the corresponding WKB spectral density $\nu_{W K B}$

$$
\nu_{W K B}(e)=\int_{-\infty}^{y_{1}} d y \frac{1}{2 \pi \sqrt{e-v(y)}}
$$

where $y_{1}$ is the first root of $e-v(y) . \nu_{W K B}(e)$ can be expressed in terms of elliptic integrals. When there are 3 real roots $y_{1}<y_{2}<y_{3}$ of $v(y)-e=0$, from eqs. (3.131.1) and (8.112.1) of Ref. 25] we obtain

$$
\nu_{W K B}(e)=\frac{1}{\pi \sqrt{y_{3}-y_{1}}} \mathbf{K}(p) ; \quad p=\sqrt{\frac{y_{3}-y_{2}}{y_{3}-y_{1}}}
$$

When there is one real root $y_{1}$ and two complex-conjugate roots $y_{2}^{*}=y_{3}$, one can use eq. (8.126.1) of Ref. 25] to transform (32) into the form

$$
\nu_{W K B}(e)=\frac{1}{\pi \sqrt{\eta}} \mathbf{K}(\sin (\phi / 2)) ; \quad y_{2}-y_{1}=\eta e^{i \phi}
$$

The "Fermi energy" corresponding to the WKB solution, $e_{F}^{W K B}$ is defined by

$$
1=\int_{-\infty}^{e_{F}^{W K B}} \operatorname{de} \nu_{W K B}(e)
$$

Outside the scaling region the exact spectral density is equal to the WKB density,

$$
\nu(e)=\nu_{W K B}(e) \quad \text { for } \quad e<e_{l o w},
$$

where $e_{\text {low }}$ is some large negative value of $e$. The WKB solution for the Fermi energy $e_{F}^{W K B}$ for $\epsilon>0$ was found in [8]. It exactly coincides with the bottom of the second well, which in our normalization is

$$
e_{F}^{W K B}=-\frac{2}{3} \epsilon^{3 / 2}
$$

Combining (29), (34), (35) and (36), we obtain the final implicit equation for the Fermi energy $e_{F}$ as determined by the scaling region:

$$
\int_{e_{\text {low }}}^{e_{F}^{W K B}} d e \nu_{W K B}(e)=\int_{e_{\text {low }}}^{e_{F}} d e \nu(e) .
$$


The exact solution for spectral density can be obtained from the Gelfand-Dikii equation 24 for the resolvent $G(e, y)$

$$
-2 G \partial^{2} G / \partial y^{2}+(\partial G / \partial y)^{2}+4(v-e) G^{2}=1
$$

Under usual circumstances, when the parameters are not fine-tuned to magnify the scaling region, the G-D equation is rather useless, as the direct solution of the Schrödinger equation is simpler. However, in our case it is just what we need. Differentiating (38) with respect to $y$, dividing by $G$ and taking the imaginary part, we obtain an ordinary third-order linear differential equation for the continuous particle density $\rho(e, y)$ in the double scaling limit,

$$
\rho^{\prime \prime \prime}=2 v^{\prime} \rho+4(v-e) \rho^{\prime}
$$

At large $|y|$, the general asymptotic form of the solution for $\rho$, correct up to terms $\sim \mathcal{O}(1 /|v|)$, can be written in terms of three integration constants $c_{1}^{ \pm}, c_{2}^{ \pm}$and $c_{3}^{ \pm}$:

$$
\rho \rightarrow \frac{c_{1}^{ \pm} \exp \left(2 \int^{y} \sqrt{v} d y\right)+c_{2}^{ \pm} \exp \left(-2 \int^{y} \sqrt{v} d y\right)+c_{3}^{ \pm}}{\sqrt{|v|}} ; \quad y \rightarrow \pm \infty
$$

To chose the proper boundary conditions, physical intuition about the system must be used. At $y \rightarrow \infty$ the potential $v(y)$ grows large, so only the decaying exponential is left, while $c_{1}^{+}=c_{3}^{+}=0$. This leaves one free parameter, $c_{2}^{+}$, the overall normalization of $\rho$, which can be determined as follows. We start from the asymptotic solution (40) at some large positive $y=y_{0}$, with $c_{1}^{+}=c_{3}^{+}=0$ and some arbitrary initial value of $c_{2}^{+}$. We then solve the differential equation (39) numerically, evolving down to large negative values of $y$, where $v(y)<0$ and $\sqrt{v}$ is complex. The solution there is of the form (40), with $c_{1}^{-}, c_{2}^{-}, c_{3}^{-} \neq 0$, i.e. it contains two oscillating exponentials, plus a powerlike term. The oscillations represent a pure quantum effect, invisible in WKB expansion. If we average $\rho \sqrt{(e-v)}$ over these oscillations, the $c_{1}^{-}$and $c_{2}^{-}$terms disappear and we should obtain $\frac{1}{2 \pi}$, according to the WKB solution. This means that the solution ought to be multiplied by a constant such that $c_{3}^{-}=1 / 2 \pi$. This is the missing normalization condition for the density.

In practice averaging over the oscillations is rather tricky, as it involves delicate cancellations between the positive and the negative contributions. There is, however, a better way of extracting $c_{3}^{-}$from $\rho$. If $\rho$ is given by (40), then, up to terms $\sim \mathcal{O}(1 /|v|)$,

$$
c_{3}^{-}=\sqrt{e-v}\left(\rho-\frac{\rho^{\prime \prime}}{4(e-v)}\right)
$$

The prescription (41) has the advantage that it is local in $y$ and only requires the knowledge of $\rho^{\prime \prime}$, which is readily available in any code used for solving differential equations. The reliability of (41) can easily be tested, by verifying that the result for $c_{3}^{-}$is independent of $y$.

We employed the differential equation solving routine ODE, described in detail in Ref. [26]. The routine is very stable and extremely easy to use. It is based on a variable-step, variableorder Adams method (explicit linear multistep method). The variable-step feature is essential, because of the rapid crossover from smooth to oscillatory behavior. 
Once $\rho(e, y)$ is known, $\nu(e)$ is in principle given by (28). In practice, it is more efficient to solve for the integral of $\rho(e, y)$ :

$$
\xi(e, y)=-\int_{y}^{\infty} d y \rho(e, y) ; \quad \rho(e, y)=\partial \xi(e, y) / \partial y
$$

which satisfies the 4-th order differential equation

$$
\xi^{\prime \prime \prime \prime}=2 v^{\prime} \xi^{\prime}+4(v-e) \xi^{\prime \prime}
$$

yielding both $\rho(e, y)$ and $\nu(e)$ at the same time,

$$
\nu(e)=-\lim _{y \rightarrow-\infty} \xi(e, y)
$$

The boundary conditions for the derivatives of $\xi$ are those for $\rho$ and its derivatives. In addition, $\xi\left(y_{0}, e\right)=0$, where $y_{0}$ is large and positive.

While solving eq. (43) for the various values of $e$, we store the values of $\rho(e, y)$ on a twodimensional grid in the $[e, y]$ plane. These are to be used later in obtaining the distribution of the eigenvalues of a random matrix (see eq. (45) below).

The tail of the integral in (42) converges rather slowly, since $\rho(e, y) \sim|y|^{-3 / 2}$ for large negative $y$. In that region, however, the integral of $\rho(e, y)$ is very well approximated by the integral of $\rho_{W K B}(e, y)$, eq. (30). The corresponding WKB tail $\tau(y)$ can expressed in terms of elliptic integrals, which we calculated numerically using standard methods.

The solution of the G-D equation for $\rho$ at $\epsilon=1$ and $e=-\frac{2}{3}$, which corresponds to the bottom of the second well is presented in Fig. 1. The quantum effects are huge!

Once $\nu(e)$ is known, we obtain the Fermi energy $e_{F}(\epsilon)$ from eq. (37). The result is shown in Fig. 2.

We can now obtain the distribution $\Omega_{\epsilon}(y)$ of the eigenvalues of a random matrix in the double scaling limit. This is done by integrating $\rho(e, y)$ with respect to $e$, up to $e_{F}(\epsilon)$ :

$$
\Omega_{\epsilon}(y)=\int_{-\infty}^{e_{F}(\epsilon)} d e \rho(e, y)
$$

In practice the integrand is obtained by interpolating $\rho(e, y)$ from the previously stored twodimensional grid in the $[e, y]$ plane. The resulting $\Omega_{\epsilon}(y)$ is shown in Fig. 3. and compared with the corresponding WKB density,

$$
\Omega_{\epsilon}^{W K B}(y)=\int_{-\infty}^{e_{F}^{W K B}(\epsilon)} d e \rho_{W K B}(e, y)=\frac{1}{\pi} \sqrt{e_{F}^{W K B}-v(y)}
$$

The contribution of the non-perturbative effects in physical observables can most easily be seen by computing loop averages for positive $l$ :

$$
W_{\epsilon}(l)=\frac{1}{Z_{\epsilon}} \int_{-\infty}^{\infty} d \eta \Omega_{\epsilon}(\eta) e^{l \eta}
$$

where

$$
Z_{\epsilon}=\int_{-\infty}^{\infty} d \eta \Omega_{\epsilon}(\eta)
$$


The integral in (47) converges fast on both ends. For large positive $y$, one is in the classically forbidden region, where $\Omega_{\epsilon}$ decreases faster than exponentially (see Fig. 3), and for large negative $y$ the exponential factor in the integrand ensures exponential convergence. Thus in practice it turns out to be sufficient to take a finite upper limit of the integration at $y=y_{\text {right }}$, i.e. the value of $y$ at which $\Omega_{\epsilon}(y)$ becomes sufficiently small.

Comparison of $W_{\epsilon}(l)$ with its WKB analogue, defined by eqs. (47), (48) with $\Omega_{\epsilon}^{W K B}(y)$ instead of $\Omega_{\epsilon}(y)$, shows that the non-perturbative part of spectral density takes over the WKB part for large $l$. The results for $W_{\epsilon}(l)$ for $0<l<6$ are shown in Fig. 4.

Note, that the exact loop average, unlike the WKB one, reaches the minimum, and then grows! This is a direct consequence of the fact that the scaling eigenvalue density $\Omega_{\epsilon}(y)$ is different from zero in the classically forbidden region $y>0$.

In physical terms, this striking phenomenon is explained as follows. The larger the boundary $l$ of our two dimensional space, the more area it could encircle, and hence, the more handles could be attached to it. The number of surfaces with unlimited genus grows as exponential of the fifth power of the area [7], and a typical area grows as the square of the length of its boundary. So, we might expect a very fast growth of the loop average due to nonperturbative contributions.

Another comment: in the literature, doubts were raised [27], whether the Marinari-Parisi prescription (in the WKB approximation) could preserve the positivity of the loop average. We did not observe such pathologies. Moreover, we believe that with correct nonperturbative definition of the loop average the positivity is guaranteed.

The point is that the spectrum of the random matrix in the Marinari-Parisi model is infinite, it covers the whole real axis. Our spectral integral for the loop average is manifestly positive definite.

However, in the WKB approximation, studied in [27], there are several disconnected regions of the classically allowed motion with gaps in between. Our prescription is to add all these contributions with positive sign. Being rewritten as a contour integral, this would produce a set of loops encircling each allowed region anticklockwise, or a single contour going in the imaginary direction to the right of all the regions.

For positive $l$ one can close the contour in the left half plane, yielding the original spectral integral. For negative $l$ one could close the contour in the right half plane, yielding zero, as there are no singularities of the integrand.

\section{Acknowledgements}

This research was supported in part by grant No. 90-00342 from the United StatesIsrael Binational Science Foundation (BSF), Jerusalem, Israel, and by the Basic Research Foundation administered by the Israel Academy of Sciences and Humanities. One of us (A.M.) would like to thank the Institute for Advanced Study at the Tel-Aviv University for hospitality. This work was partially supported by the National Science Foundation under contract PHYS-90-21984. 


\section{References}

[1] E. Brezin and V. A. Kazakov, Phys. Lett. B236 (1990) 144; M. R. Douglas and S. H. Shenker, Nucl. Phys. B335 (1990) 635; D. J. Gross and A. Migdal, Phys. Rev. Lett. 64 (1990) 127.

[2] A. Migdal, Phys. Rep. 102 (1983) 201; V. Kazakov, Phys. Lett. B150 (1985) 282; F. David, Nucl. Phys. B257 (1985) 45; V. Kazakov, I. Kostov and A. Migdal, Phys. Lett. B157 (1985) 295.

[3] D. J. Gross and A. Migdal, Phys. Rev. Lett. 64 (1990) 127; M. Douglas, Phys. Lett. B238 (1990) 176; T. Banks, M. R. Douglas, N. Seiberg and S. H. Shenker, Phys. Lett. B238 (1990) 279.

[4] E. Brezin, E. Marinari and G. Parisi Phys. Lett. B242 (1990) 35.

[5] F. David, Mod. Phys. Lett. A5 (199) 1019.

[6] M. R. Douglas, N. Seiberg and S. H. Shenker Phys. Lett. B244 (1990) 381.

[7] D. J. Gross and A. Migdal, Nucl. Phys. B340 (1990) 335.

[8] E. Marinari and G. Parisi, Phys. Lett. B240 (1990) 375. Phys. Lett. B247 (1990) 537.

[9] J. Greensite, M. B. Halpern, Nucl. Phys. B242 (1984) 167.

[10] M. Karliner and A. Migdal, Mod. Phys. Lett. A5 (1990) 2565.

[11] J. Ambjørn, J. Greensite and S. Varsted, Phys. Lett. B249 (1990) 411.

[12] J. Ambjorn and J. Greensite, Phys. Lett. B254 (1991) 66.

[13] J. Gonzalez, Phys. Lett. B255 (1991) 367.

[14] S. Dalley, C. Johnson and T. Morris, Nucl. Phys. B368 (1992) 655; S. Dalley, C. Johnson and T. Morris, Non-Perturbative Two-Dimensional Quantum Gravity, Again, hepth/9108016.

[15] J. Ambjørn, C. Johnson and T. Morris, Nucl. Phys. B374 (1992) 496.

[16] J. Gonzalez, M.A.H. Vozmediano, Phys. Lett. B258 (1991) 55.

[17] J. Feinberg, Phys. Lett. B281 (1992) 225; Stabilized Matrix Models for Nonperturbative Two Dimensional Quantum Gravity, preprint TECHNION-PH-35, Dec. 1992, hep-th/9212069.

[18] J. L. Miramontes, J. S. Guillen, Int. J. Mod. Phys. A7 (1992) 6457.

[19] J. Greensite, Stabilized Quantum Gravity: Stochastic Interpretation and Numerical Simulation, preprint SFSU-TH-92-1, Apr. 1992, hep-th/9205006. 
[20] J. Ambjorn and C.F. Kristjansen, Nonperturbative 2-D Quantum Gravity and Hamiltonians Unbounded From Below, preprint NBI-HE-92-28, May 1992, hep-th/9205073.

[21] J. Greensite, Phys. Lett. B291 (1992) 405.

[22] A. V. Turbiner, Sov. Phys. Usp. 27 (1984) 668, [Usp. Fiz. Nauk 144 (1984) 35 ].

[23] E. Brezin, M. R. Douglas, V. A. Kazakov and S. H. Shenker Phys. Lett. B237 (1990) 43 ;

D. J. Gross and A. Migdal, Phys. Rev. Lett. 64 (1990) 717;

C. Crnković, P. Ginsparg and G. Moore Phys. Lett. B237 (1990) 196;

E. Brezin, V. A. Kazakov and Al. B. Zamolodchikov, Nucl. Phys. B338 (1990) 673.

G. Parisi, Phys. Lett. B238 (1990) 209; G. Parisi, Europhys. Lett. 11 (1990) 595;

D. J. Gross and N. Miljković, Phys. Lett. B238 (1990) 217; P. Ginsparg and J. ZinnJustin, Phys. Lett. B240 (1990) 333.

G. Parisi, Phys. Lett. B238 (1990) 213.

[24] I. Gelfand and L.Dikii, Usp. Mat. Nauk 30 (1975) 5.

[25] I. S. Gradshteyn and I. M. Ryzhik, Table of Integrals, Series, and Products, Academic Press, 1965.

[26] L. F. Shampine and M. K. Gordon, Computer Solution of Ordinary Differential Equations: The Initial Value Problem, Freeman, 1975.

[27] F. David, Nonperturbative Effects in Two-Dimensional Quantum Gravity, SACLAYSPHT-91-114, Dec 1990 and Proc. 8-th Jerusalem Winter School for Theoretical Physics, Two-Dimensional Gravity and Random Surfaces, Jerusalem, Israel, Dec. 27 - Jan. 4, 1991, and Nonperturbative Effects in 2-D Gravity and Matrix Models, SACLAY-SPHT90-178, May 1990, in Proc. Cargese Workshop 'Random Surfaces, Quantum Gravity and Strings', Cargese, France, May 27 - Jun 2, 1990. 


\section{Figure Captions}

- Figure 1. The particle density $\rho(e, y)$, solution of eq. (39), for $\epsilon=1$ and $e=-\frac{2}{3}$. Dash-dotted line denotes the WKB solution.

- Figure 2. The Fermi energy, $e_{F}(\epsilon)$, obtained from eq. (37). The diamonds denote the actual values computed, the continuous curve is plotted to guide the eye. Dash-dotted line denotes the Fermi energy in the WKB approximation, $e_{F}^{W K B}(\epsilon)$.

- Figure 3. The scaling eigenvalue density $\Omega_{\epsilon}(y)$. The WKB approximation is plotted as a dash-dotted line.

a) $\Omega_{\epsilon}(y)$ and $\Omega_{\epsilon}^{W K B}(y), \epsilon=-1,0,1$.

b) $\Delta \Omega_{\epsilon}(y)=\Omega_{\epsilon}^{W K B}(y)-\Omega_{\epsilon}(y), \epsilon=-1,0,1$.

- Figure 4. Loop average $W_{\epsilon}(l)$, eq. (47), for $\epsilon=-1,0,1$. The WKB approximation is plotted as a dash-dotted line. 


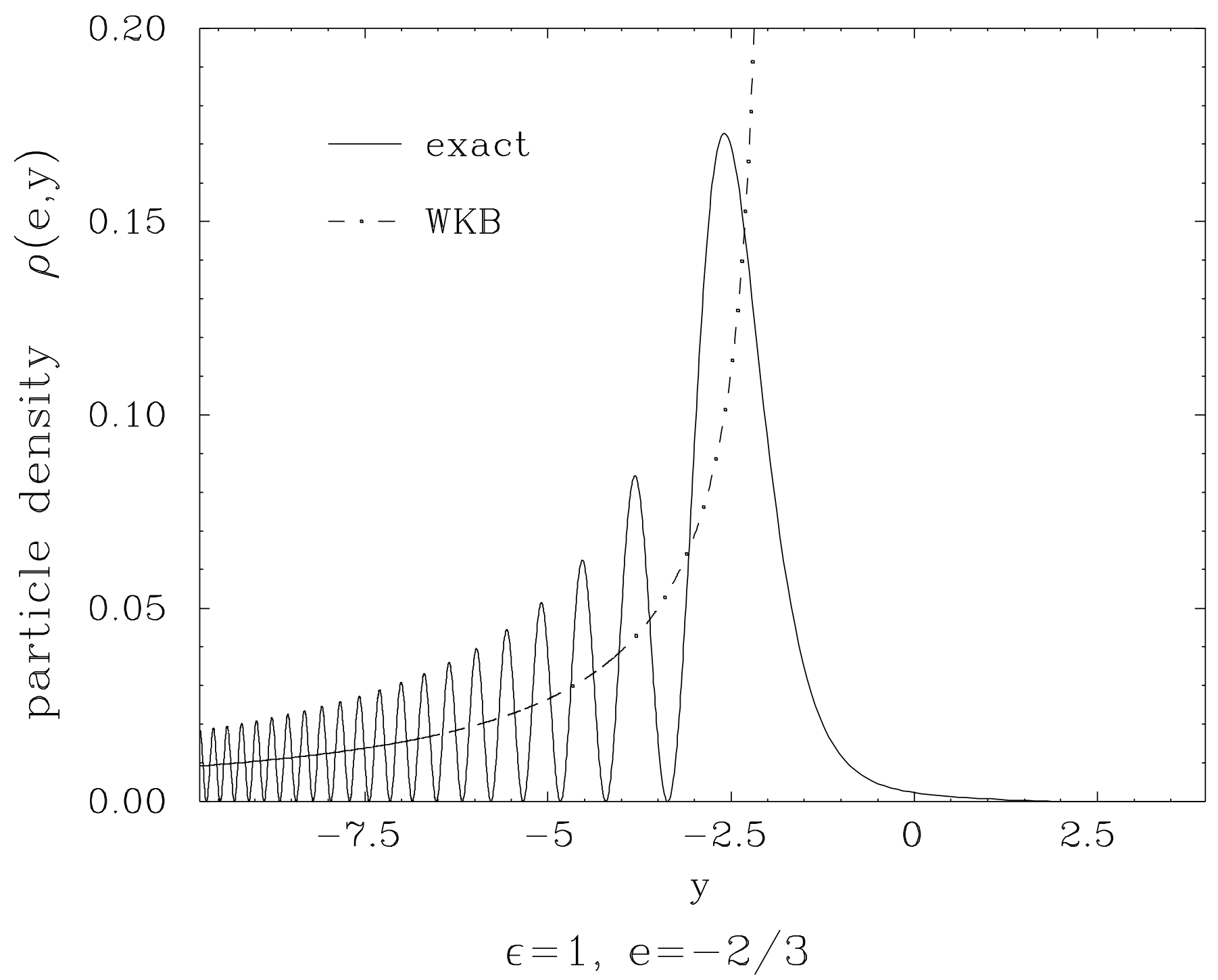




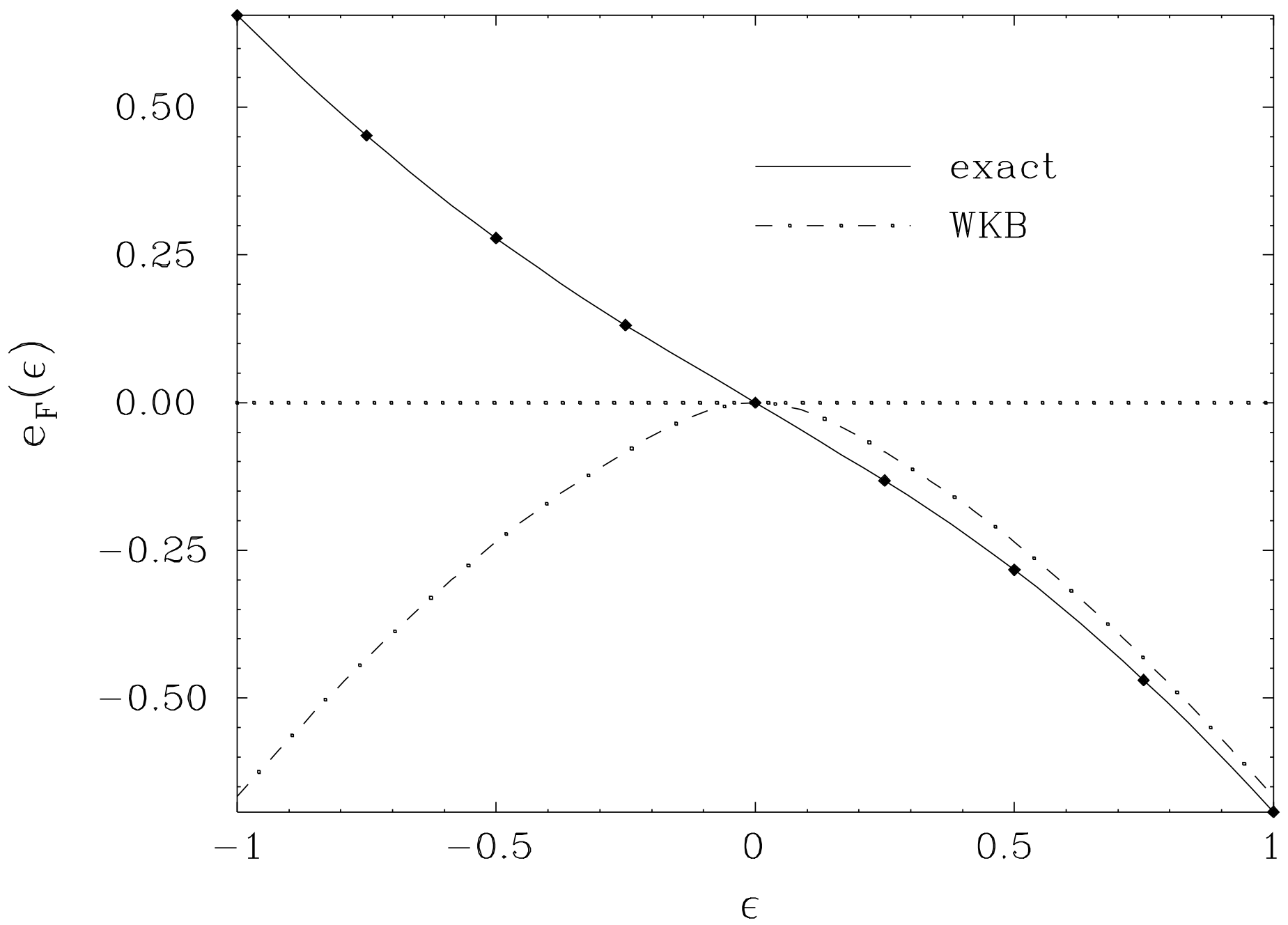


Eigenvalue density
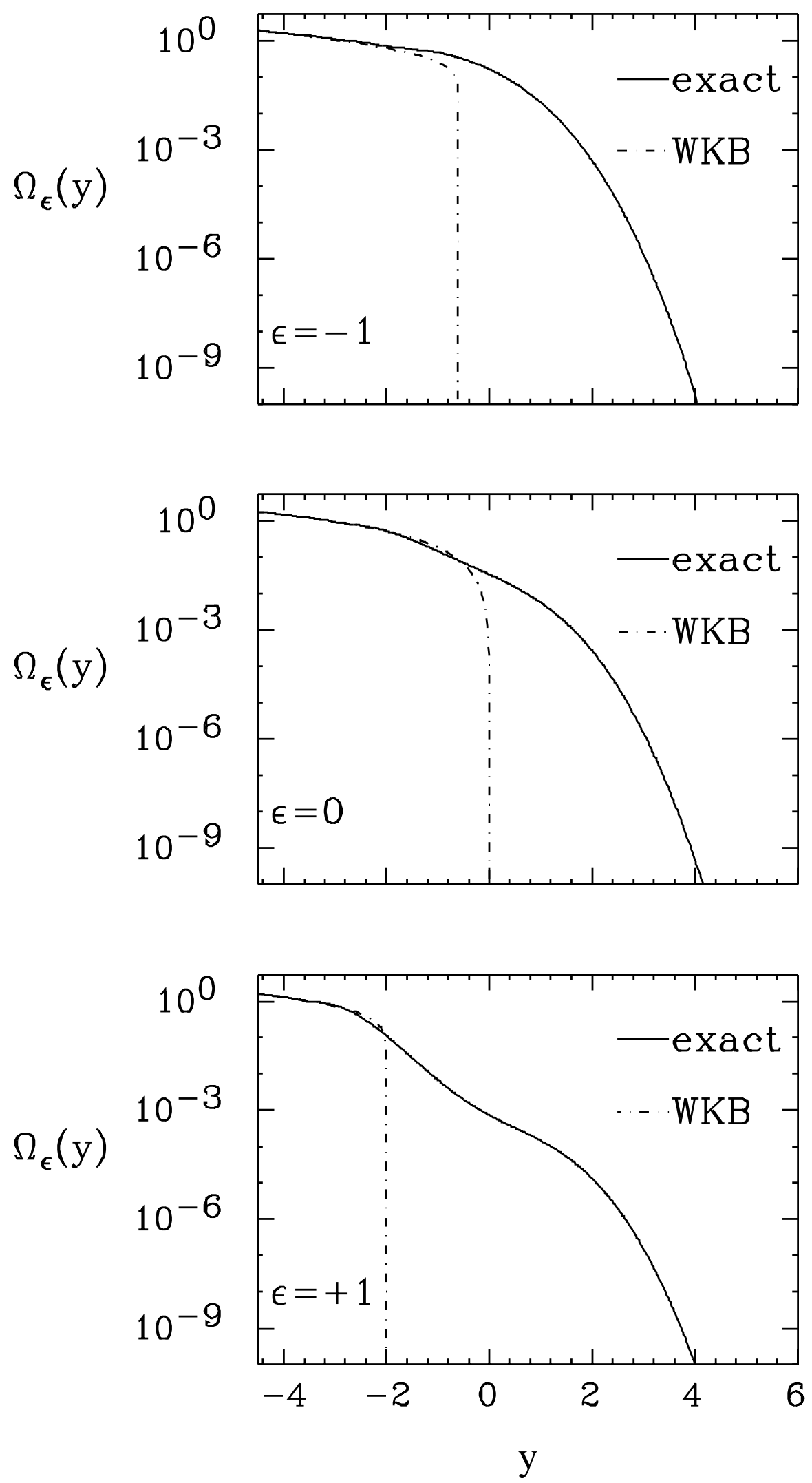


$$
\Delta \Omega(\mathrm{y})=\Omega_{\mathrm{wKB}}(\mathrm{y})-\Omega_{\text {exact }}(\mathrm{y})
$$
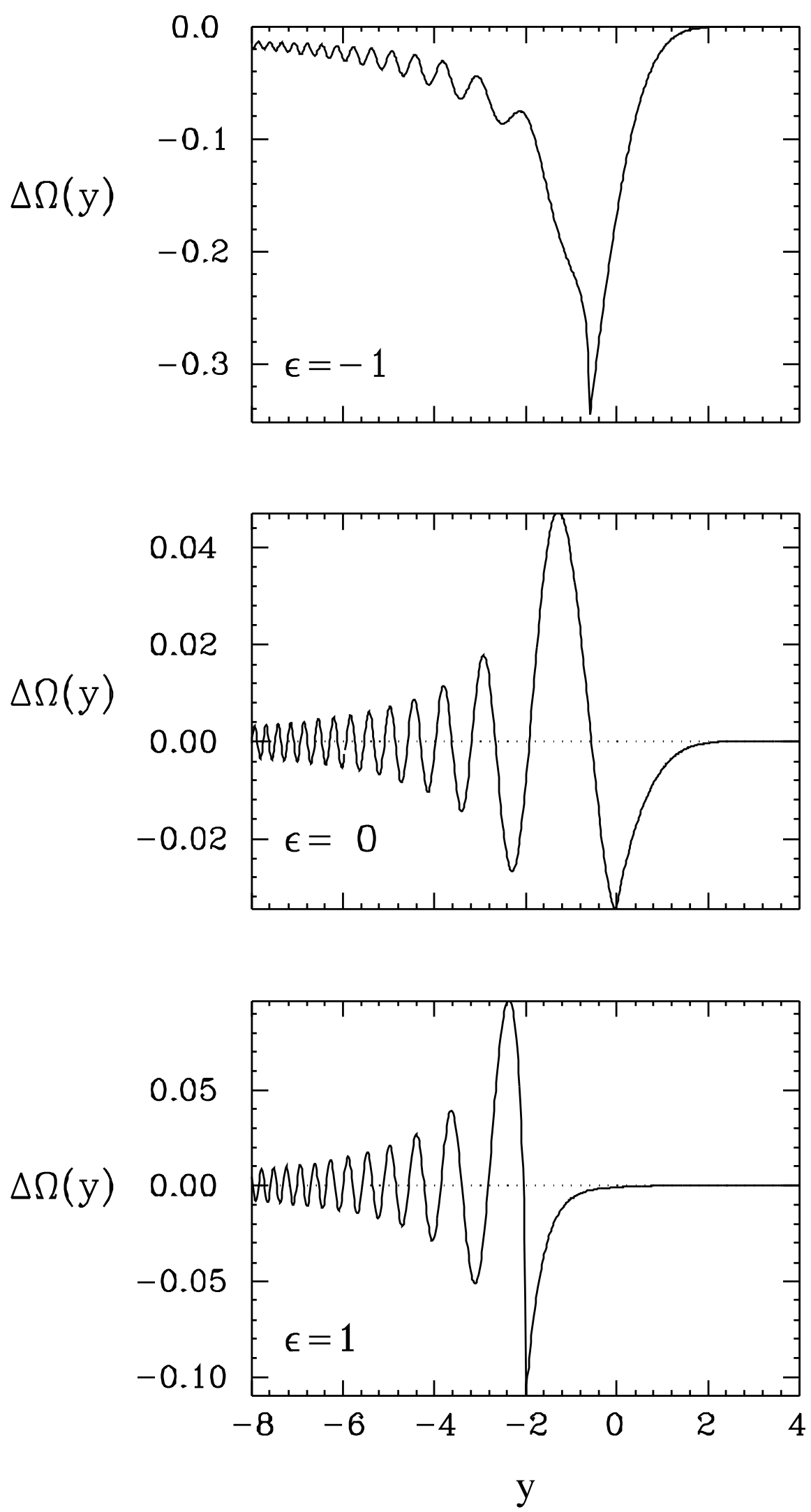
Loop average $<\mathrm{W}(\mathrm{l})>$
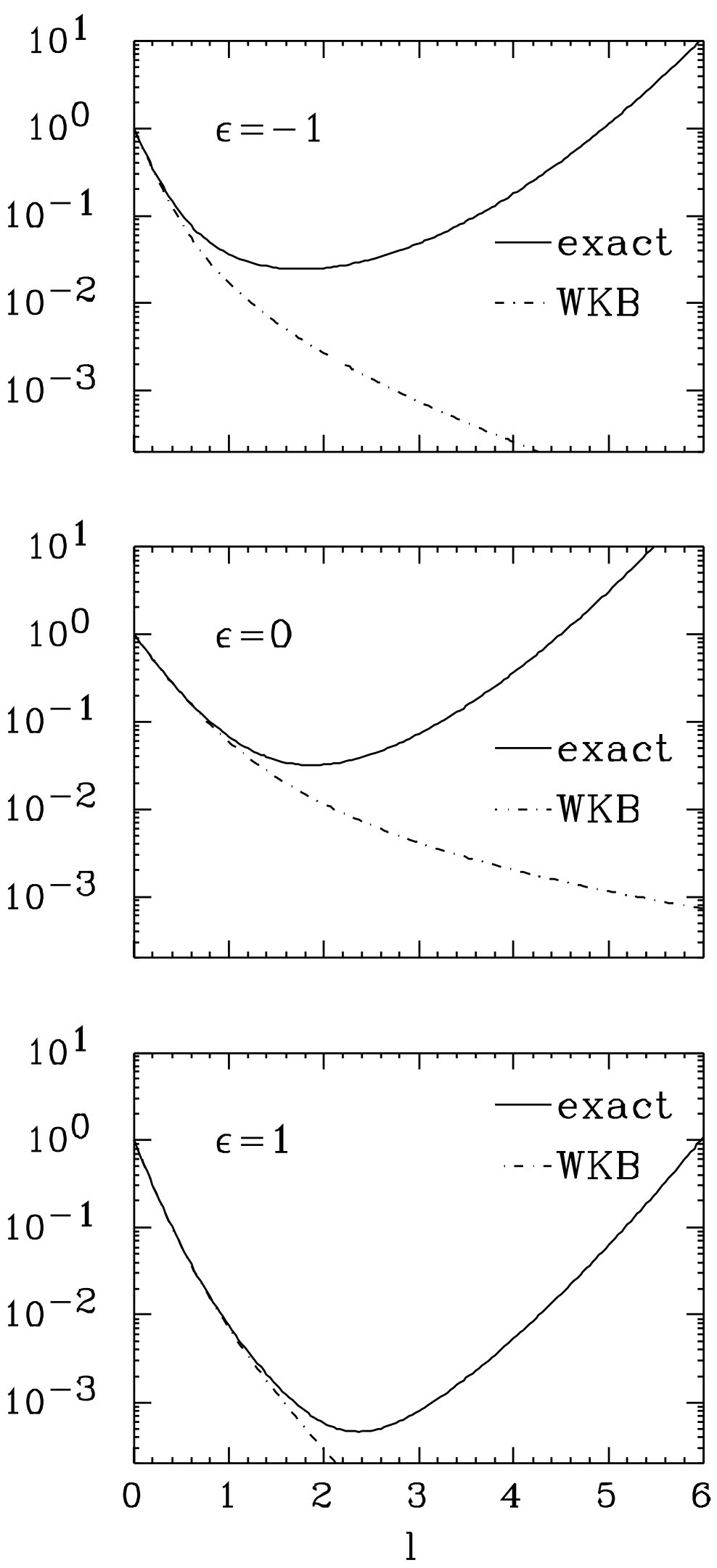